\title{
Absceso periostomal secundario a perforación intestinal por cuerpo extraño: presentación de un caso
}

\author{
Christophe Riquoir A. ${ }^{1}$, Eugenio Grasset E. ${ }^{2,3}$ y Felipe Bellolio R. ${ }^{2}$
}

\section{Periostomal abscess secondary to bowel perforation due to foreign} body ingestion: A case report

\begin{abstract}
Aim: To report a case of periostomal abscess, its clinical and imaging diagnosis and management. Materials and Method: 77-year-old patient, user of a colostomy with abdominal pain associated to swelling and redness of the skin next to the colostomy. A computed tomography of the abdomen showed a foreign body perforating the ostomized bowel associated to the formation of an abscess. Results: An incision of the gathering was performed, giving out a great quantity of pus and the foreign body, which corresponded to a bird's tibia. Antibiotic therapy was given, and a Penrose drainage installed. Further controls showed regression of the abscess and no flux from drainage. Discussion: $80 \%-90 \%$ of ingested foreign bodies are eliminated without complications and $<1 \%$ produce perforation. This is more common in angled intestinal segments or surgically intervened ones. Clinical features are unspecific, and diagnosis requires suggesting imaging signs. Conclusion: Periostomal abscesses and bowel perforation due to foreign body are infrequent. High diagnostic suspicion and an imaging evaluation may give a precise answer. Besides surgical management, antibiotic coverage for enteropathogens must be associated and a drainage system too in most cases.
\end{abstract}

Key words: ostomy; abscess; foreign body.

\section{Resumen}

Objetivo: Reportar un caso de absceso periostomal, su diagnóstico clínico e imagenológico y manejo. Materiales y Método: Paciente de 77 años usuaria de colostomía con dolor abdominal asociado a aumento de volumen y enrojecimiento de la piel alrededor de la colostomía. Una tomografía computada de abdomen muestra un cuerpo extraño perforante de la pared colónica ostomizada, asociado a formación de un absceso. Resultados: Se practica una incisión de la colección, dando salida a gran cantidad de pus y cuerpo extraño correspondiente a tibia de ave. Se inicia cobertura antibiótica y se instala drenaje Penrose. Controles posteriores muestran regresión del absceso y drenaje sin débito. Discusión: $80 \%-90 \%$ de los cuerpos extraños ingeridos son eliminados sin complicación $\mathrm{y}<1 \%$ producen perforación. Esto es más común en segmentos intestinales angulados o intervenidos quirúrgicamente. La clínica es inespecífica y el diagnóstico requiere una imagen que identifique signos sugerentes. Conclusión: Un absceso periostomal y la perforación intestinal por cuerpo extraño son cuadros infrecuentes. La alta sospecha diagnóstica y una evaluación imagenológica pueden dar una respuesta precisa. Además del manejo quirúrgico, debe asociarse cobertura antibiótica para enteropatógenos y generalmente un sistema de drenaje.

Palabras clave: ostomía; absceso; cuerpo extraño.
'Escuela de Medicina,

Pontificia Universidad Católica de Chile. Santiago, Chile. ${ }^{2}$ Departamento de Cirugía Digestiva, Pontificia Universidad Católica de Chile. Santiago, Chile.

${ }^{3}$ Departamento de Cirugía

Oriente, Universidad de Chile. Santiago, Chile.

Recibido 2019-03-17 y aceptado 2019-06-19

Correspondencia a: Dr. Christophe Riquoir Altamirano cfriquoir@uc.cl

\section{Introducción}

La incidencia global de complicaciones de la ostomía y de la piel periostomal se estima en alrededor de $50 \%^{1}$. Las más comúnmente descritas son: re- tracción, hernias, prolapsos, necrosis y alteraciones de la piel periostomal ${ }^{2}$. En el período posoperatorio temprano, las infecciones y abscesos periostomales son relativamente raras, con una incidencia reportada entre $2 \%$ y $14,8 \%{ }^{3}$. Esto se ve con mayor 
frecuencia en cirugías de revisión de la ostomía, debido principalmente a la colonización preoperatoria de la piel periostomal y a la inoculación bacteriana en el perioperatorio. En los casos de presentación tardía, se asocia principalmente a foliculitis local o a recurrencia de alguna enfermedad inflamatoria intestinal ${ }^{4}$.

Por otra parte, las principales causas de perforación de intestino grueso son tumores, diverticulitis, infecciones o abscesos y colitis, mientras que causas infrecuentes son: cuerpo extraño, obstrucción, iatrogénica y trauma ${ }^{5}$. La perforación intestinal por cuerpo extraño se debe a la ingesta, generalmente inadvertida, de elementos de la dieta, en especial huesos de pescado u otro animal, dientes y mondadientes. Debido a lo anterior, es infrecuente contar con una historia clara de la ingesta del cuerpo extraño $^{6,7}$

A continuación, se presenta el caso de una paciente que presentó un absceso periostomal secundario a una perforación intestinal por cuerpo extraño.

\section{Caso clínico}

Paciente de 77 años, sexo femenino, con antecedente de una exanteración total pelviana asociada a colostomía terminal y ureterostomía tipo Bricker realizada en 2013 debido a un carcinoma pélvico indiferenciado sobreinfectado, fistulizado a recto, vagina y vejiga.

Consulta seis años después en el policlínico por un cuadro de tres días de evolución caracterizado por dolor intenso, eritema y aumento de volumen de la piel alrededor de la colostomía en su borde inferolateral. No presenta fiebre, compromiso de conciencia, náuseas, vómitos ni otros síntomas. Sin cambios del tránsito intestinal. En el laboratorio destaca una proteína $\mathrm{C}$ reactiva en $8,39 \mathrm{mg} / \mathrm{dL}(\mathrm{VN}$ : $<1)$ y 7.500 leucocitos/uL. Se rescata una tomografía computada (TC) de hace siete días solicitada en contexto de seguimiento de neoplasia que no informa signos de complicación de la ostomía. No presenta otras alteraciones en el estudio inicial.

Al examen físico se evidencia un aumento de volumen sensible, enrojecido, indurado no fluctuante (Figura 1). Se complementa el estudio con una ecoscopía de la pared abdominal periostomal que muestra signos inflamatorios de la piel, pero no encuentra colecciones ni otros signos de complicación. Dado lo anterior se sospecha una celulitis de pared abdominal y se maneja con analgesia y esquema antibiótico con amoxicilina-ácido clavulánico por 10 días.
La paciente persiste con dolor y presenta mayor induración con cambios de coloración del cuadrante inferior izquierdo periostomal. Se controla con una nueva tomografía computada que identifica un cuerpo extraño de densidad ósea y morfología alargada, de base ancha con ahusamiento distal, cuyo tercio proximal se encuentra dentro de la porción extraabdominal del colon ostomizado. Éste atraviesa la pared colónica hacia el tejido celular subcutáneo, donde se encuentra rodeado por una colección de paredes anfractuosas que mide $4,0 \times 2,5 \times 2,8 \mathrm{~cm}$ en sus ejes mayores, compatible con absceso. Se describe también una hernia paraostomal en relación con la ureterostomía tipo Bricker en flanco derecho, que contiene parte del colon transverso y en cuyo lumen se observa otro cuerpo extraño, pero sin signos de complicación (Figura 2). Además, se encuentran dos otros cuerpos extraños en el marco colónico de similares características, para los cuales se desestimó necesidad de intervención (Anexo 1). La paciente refiere no tener recuerdo de haberlos ingerido.

Se practicó un drenaje abierto del absceso en pabellón con una incisión sobre la colección fuera del borde de la placa de ostomía, obteniendo abundante salida de material purulento. A la exploración se logra extraer el cuerpo extraño, que resulta ser un tibia de ave (Figura 3). Dada la profundidad del defecto y estrechez del lumen ostomal se decide no reparar el defecto en la pared del colon a la espera de su cicatrización espontánea. Se instala un drenaje

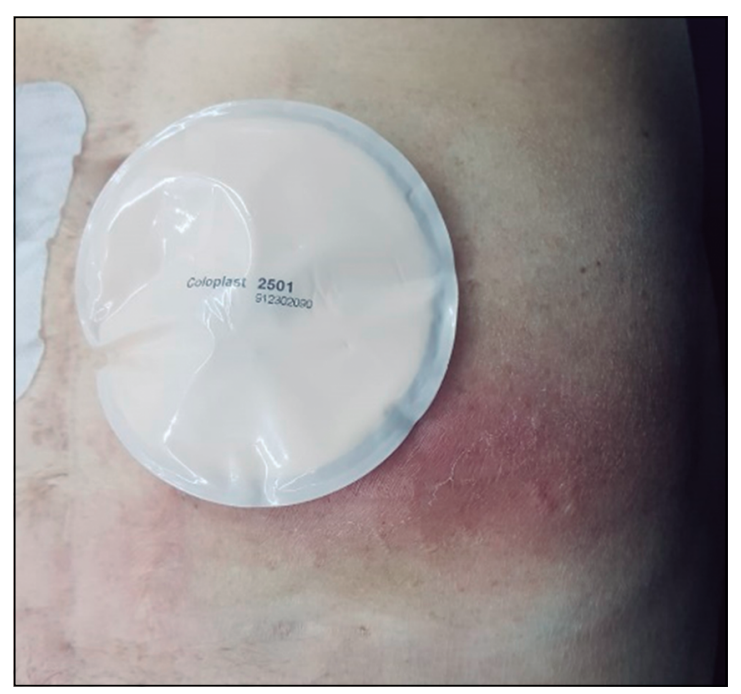

Figura 1. Hallazgos al examen físico. Se puede apreciar enrojecimiento y aumento de volumen en el borde inferolateral de la ostomía. 

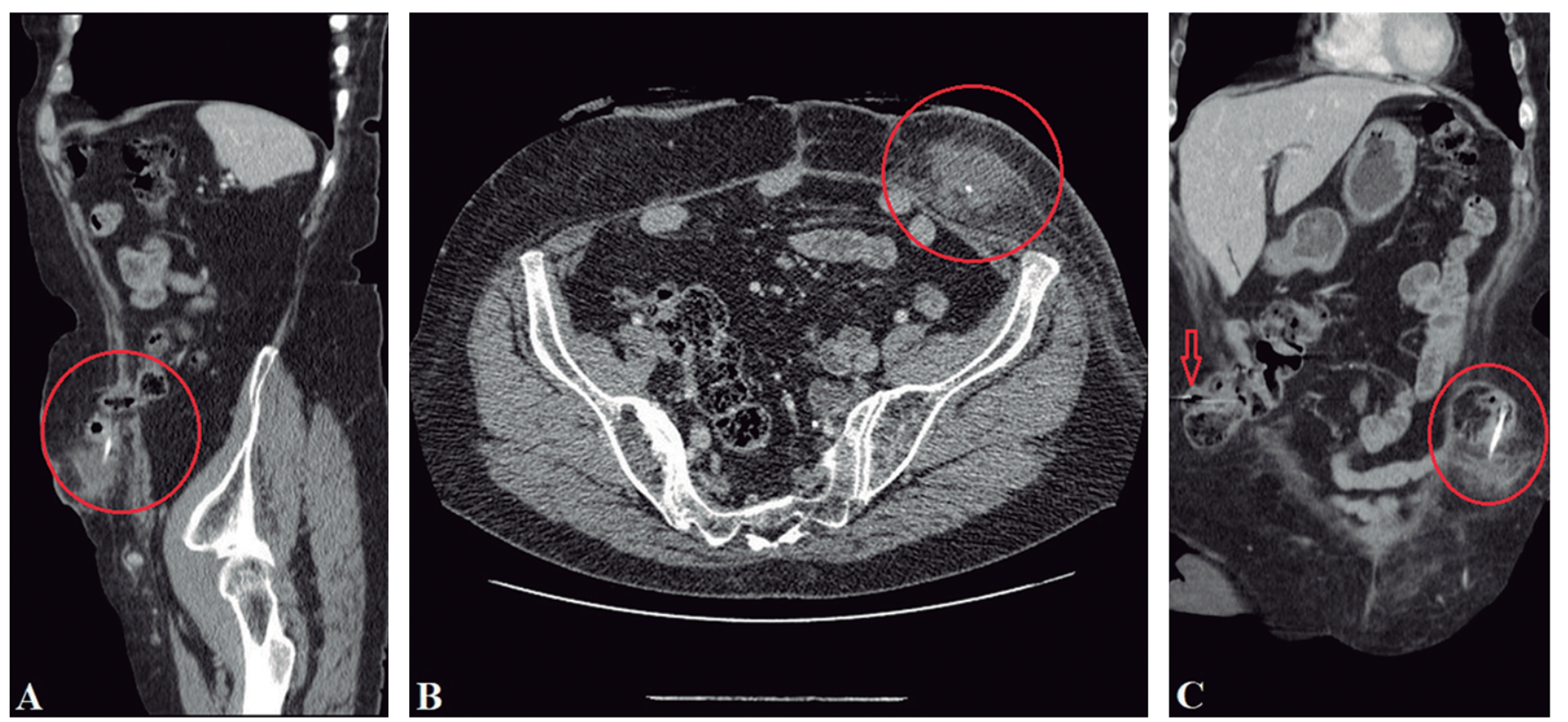

Figura 2. Tomografía computada de abdomen y pelvis: se identifica en la imagen $\mathbf{A}$ un corte sagital de la salida de la colostomía y un cuerpo extraño hiperdenso en la pared intestinal con salida hacia el tejido subcutáneo y en contacto con un absceso. La imagen B muestra un corte transversal donde se observa el absceso en relación con la pared abdominal y el cuerpo extraño en contacto. La imagen $\mathbf{C}$ muestra un corte coronal en el que se puede apreciar una mayor extensión del cuerpo extraño (círculo). Se observa además, en flanco derecho, una hernia paraostomal con asas colónicas, en cuyo lumen se identifica otra imagen hiperdensa sugerente de cuerpo extraño, pero sin signos de complicación (flecha).
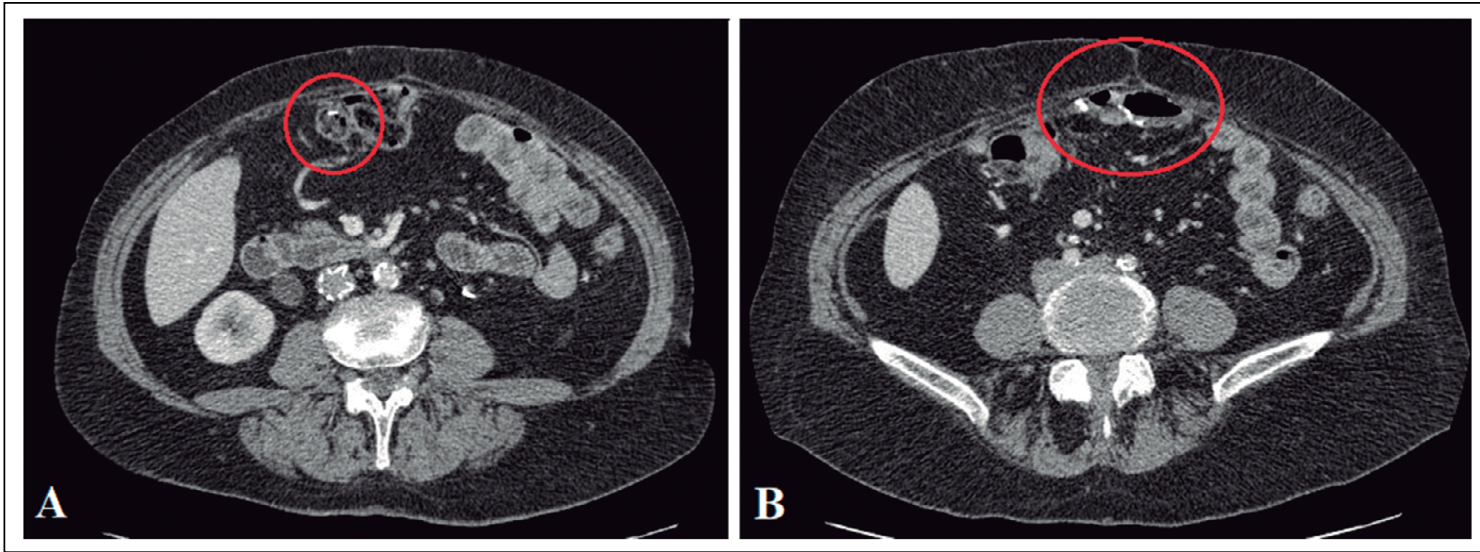

Anexo 1. En ambas imágenes se pueden observar cuerpos extraños intraluminales $(\mathbf{A})$ y en estrecha relación con la pared intestinal (B), ambos sin signos de complicación.

Penrose en el sitio de la incisión y se realiza recambio de la bolsa de ostomía. La paciente evoluciona de manera favorable, siendo dada de alta a las $48 \mathrm{~h}$ con indicación de continuar tratamiento antibiótico vía oral por 4 días con ciprofloxacino y metronidazol. Al décimo día posoperatorio se realiza retiro del drenaje en la consulta de curaciones con plan de cierre por segunda intención de la herida operatoria (Figura 4).

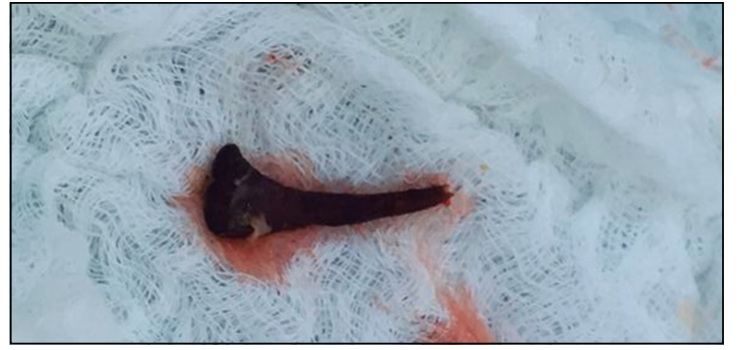

Figura 3. Cuerpo extraño extraído correspondiente a tibia de ave. 


\section{CASOS CLÍNICOS}

Figura 4. En la imagen superior se observa el posoperatorio inmediato con el drenaje Penrose in situ. En la imagen inferior se observa la evolución a los diez días tras el retiro del drenaje. La herida se encuentra abierta sin signos de sobreinfección.

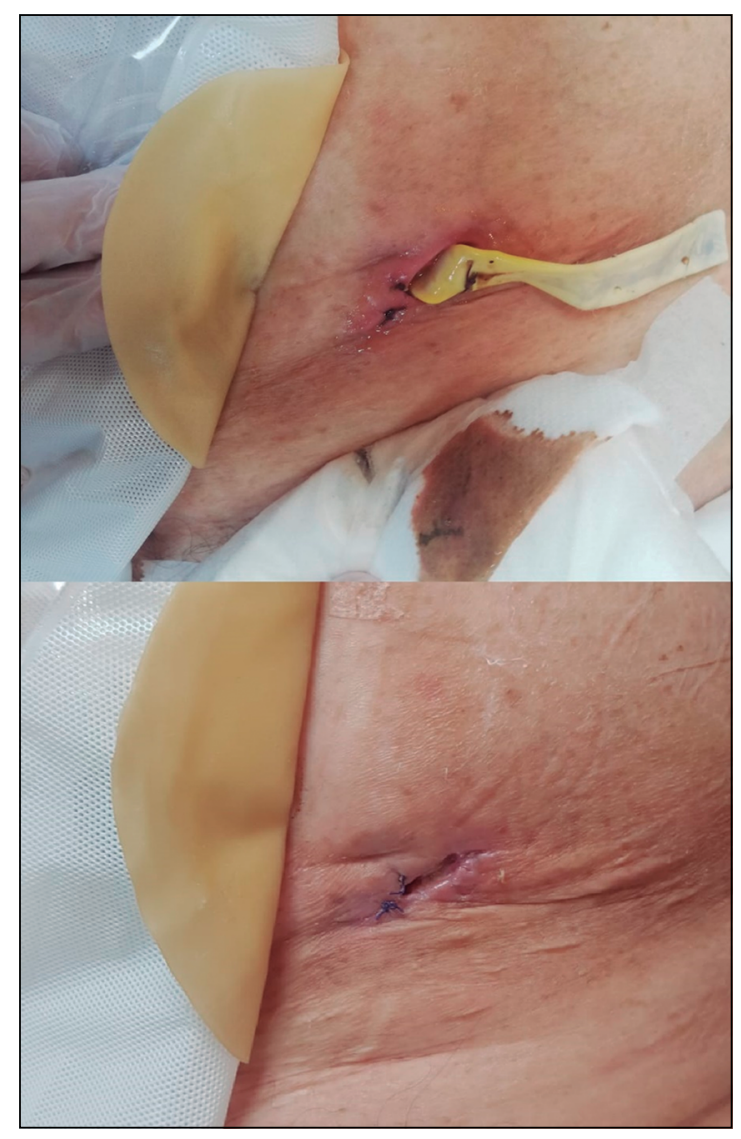

\section{Discusión}

Un $80 \%-90 \%$ de los cuerpos extraños ingeridos son eliminados espontáneamente sin producir complicaciones y menos del $1 \%$ se complican con una perforación ${ }^{8}$. Los sitios de perforación intestinal suelen ser aquellos segmentos con angulaciones agudas como la válvula ileocecal o la unión rectosigmoidea. Esto se ve favorecido cuando existen cirugías previas o áreas de estenosis9. Esta situación podría verse emulada en una ostomía, dado que su conformación reúne todas estas características. Sin embargo, este evento en particular es poco reportado en la literatura.

\section{Diagnóstico}

Los síntomas de una perforación intestinal suelen ser inespecíficos. Se describe anorexia, náuseas, vómitos, fiebre, baja de peso, dolor abdominal difuso o localizado, crónico o agudo e íleo, entre otros ${ }^{6}$. Esto suele confundirse muchas veces con cuadros agudos como apendicitis o diverticulitis cuando no se presta debida atención a la evolución más lenta que caracteriza a la perforación ${ }^{10}$.

Para un correcto diagnóstico de la presencia, sitio y causa de una perforación intestinal la tomografía computada es ampliamente superior a otros exámenes imagenológicos como la radiografía de abdomen simple o enema de doble contraste. Los hallazgos directos de perforación son aire o contraste extraluminal y una discontinuidad en la pared intestinal. Son hallazgos indirectos los abscesos o flegmones con o sin enterocolito o cuerpo extraño, un engrosamiento abrupto de la pared, hallazgo de un sitio de obstrucción y signos de peritonitis ${ }^{11}$. La identificación del sitio de perforación aumenta de un $34 \%$ a un $97 \%$ para el segmento colónico ascendente-sigmoides y de $40 \%$ a $80 \%$ para perforaciones rectales cuando los hallazgos directos a la $\mathrm{TC}$ se combinan con los indirectos ${ }^{12}$.

En el caso de una perforación por cuerpo extraño, el signo más directo sería la identificación del mismo en proximidad a aire extraluminal. Sin embargo, la erosión que produce en la pared intestinal provoca una inflamación que con el tiempo ocluye el sitio de perforación, haciendo menos frecuente el hallazgo de aire extraluminal. Otros hallazgos sugerentes son el engrosamiento de la pared intestinal, aumento de la densidad de la grasa adyacente y la formación de un absceso ${ }^{13}$.

\section{Manejo}

Una perforación intestinal por cuerpo extraño puede presentarse de manera localizada con la formación de un absceso o plastrón, o generalizada por peritonitis secundaria si ésta expone el defecto a la cavidad peritoneal. Los agentes patógenos más frecuentemente aislados en estos casos son E. coli, Streptococcus spp, Enterobacter spp., Klebsiella spp., Enterococcus spp., Pseudomona aeruginosa, Proteus spp., Staphylococcus aureus y S. epidermidis ${ }^{14}$.

Por lo general, un absceso periostomal no se resuelve espontáneamente. Su manejo consta de un drenaje quirúrgico mediante una incisión en la unión mucocutánea de la ostomía o fuera del borde de ésta, cobertura antibiótica para enteropatógenos y la posterior instalación de drenaje Penrose o similar. Si el absceso no se resuelve oportunamente, pueden agregarse infecciones cutáneas graves como fasceítis necrotizante o gangrena bacteriana, $\mathrm{y}$ a su vez complicarse a nivel sistémico hasta el shock séptico ${ }^{1,15}$.

Cuando se forma un absceso periostomal por una perforación intestinal tiende a ser un cuadro de presentación tardía y mayor complejidad, so- 
bre todo en el contexto de una hernia paraostomal estrangulada, con riesgo asociado de peritonitis y requiriendo muchas veces una laparotomía asociada a resección y/o reparación de esta. Otras complicaciones tardías de la perforación intestinal asociada a absceso son la recurrencia del mismo y las fístulas, ya sea colorrectales, colovesicales, enterovasculares o colocutáneas ${ }^{14}$.

La consecuente formación de una fístula colocutánea entre el segmento intestinal comprometido y la cavidad expuesta de la colección no es infrecuente. Puede manifestarse por la salida de contenido intestinal a través de la cavidad del absceso, con irritación cutánea secundaria. Muchas de ellas cicatrizan espontáneamente, pero las persistentes o profundas requieren resección del segmento comprometido y reconfección de una nueva ostomía en un sitio diferente para evitar la infección del sitio primario ${ }^{1,4}$.

En este caso la causa no era una hernia estrangulada, sino un cuerpo extraño, que determinó un cuadro más bien benigno sin mayor repercusión clínica y ni complicaciones graves.

\section{Conclusión}

La formación de un absceso periostomal es un cuadro poco frecuente, así como también lo es la perforación intestinal por un cuerpo extraño. Ante la frecuente ausencia de una historia clínica que oriente a la causa del cuadro, la alta sospecha clínica y una evaluación de la lesión con un examen imagenológico de alta sensibilidad como la tomografía computada pueden dar una respuesta más precisa. El manejo de este tipo de abscesos se compone de drenaje quirúrgico, cobertura antibiótica para enteropatógenos y un sistema de drenaje en la mayoría de los casos. Ante la presencia de más cuerpos extraños en otros segmentos, se debe mantener alerta ante los síntomas inespecíficos descritos para evitar una complicación mayor.

\section{Responsabilidades éticas}

Protección de personas y animales. Los autores declaran que para esta investigación no se han realizado experimentos en seres humanos ni en animales.

Confidencialidad de los datos. Los autores declaran que en este artículo no aparecen datos de pacientes.

Conflictos de interés: no hay.

\section{Bibliografía}

1. De Miguel Velasco M, Jiménez Escovar F, Parajó Calvo A. Estado actual de la prevención y tratamiento de las complicaciones de los estomas. Revisión de conjunto. Cir Esp. 2014;92:149-56.

2. Salvadalena G. Incidence of complications of the stoma and peristomal skin among individuals with colostomy, ileostomy, and urostomy: a systematic review. J Wound, Ostomy Cont Nurs. 2008;35:596-609.

3. Park JJ, Del Pino A, Orsay CP, Nelson RL, Pearl RK, Cintron JR, et al. Stoma complications: The Cook County Hospital experience. Dis Colon Rectum 1999;42:1575-80

4. Kann BR. Early stomal complications. Clin Colon Rectal Surg. 2008;21:23-30.

5. Ugwu AU, Kerins N, Malik M. Traumatic small bowel perforation in a case of a perineal hernia. J Surg Case Reports 2018;2018:1-3.

6. Goh BKP, Chow PKH, Quah H-M, Ong
H-S, Eu K-W, Ooi LLPJ, et al. Perforation of the Gastrointestinal Tract Secondary to Ingestion of Foreign Bodies. World J Surg. 2006;30:372-7.

7. Ambe P, Weber SA, Schauer M, Knoefel WT. Swallowed Foreign Bodies in Adults. Dtsch Aerzteblatt Online 2012;109(Figure 2):869-76

8. Rodríguez-Hermosa JI, Codina-Cazador A, Sirvent JM, Martín A, Gironès J, Garsot E. Surgically treated perforations of the gastrointestinal tract caused by ingested foreign bodies. Colon Dis. 2008;10:701-7.

9. Gayer G, Petrovitch I, Jeffrey RB. Foreign objects encountered in the abdominal cavity at CT. Radiographics 2011;31:40928.

10. Madrona AP, Hernández JAF, Prats MC, Riquelme JR, Paricio PP. Intestinal perforation by foreign bodies. Eur J Surg. 2000;166:307-9.

11. Furukawa A, Sakoda M, Yamasaki M, Kono N, Tanaka T, Nitta N, et al.
Gastrointestinal tract perforation: CT diagnosis of presence, site, and cause. Abdom Imaging. 2005;30:524-34.

12. Imuta $M$, Awai $K$, Nakayama $Y$, Murata $Y$, Asao C, Matsukawa T, et al. Multidetector CT findings suggesting a perforation site in the gastrointestinal tract: Analysis in surgically confirmed 155 patients. Radiat Med - Med Imaging Radiat Oncol. 2007;25:113-8.

13. Kothari K, Friedman B, Grimaldi GM, Hines JJ. Nontraumatic large bowel perforation: spectrum of etiologies and CT findings. Abdom Radiol. 2017;42:2597608.

14. Barragán C, Rueda J, Espitia E, Uriza L, Rosselli D. Perforación intestinal por espina de pescado, revisión de la literatura científica y presentación de dos casos: literature review and report of two cases. Rev Colomb Cirugía 2012;79-84.

15. Stevens DL, Bryant AE. Necrotizing soft-tissue infections. N Engl J Med. 2017;377:2253-65. 